\title{
Structural aspects of the antioxidant activity of lutein in a model of photoreceptor membranes*
}

\author{
Anna Wisniewska-Becker ${ }^{1 凶}$, Grzegorz Nawrocki#, Mariusz Duda and Witold K. Subczynski² \\ 1Department of Biophysics, Faculty of Biochemistry, Biophysics and Biotechnology, Jagiellonian University, Kraków, Poland; ${ }^{2}$ Department of \\ Biophysics, Medical College of Wisconsin, Milwaukee, WI, USA
}

It was shown that in membranes containing raft domains, the macular xanthophylls lutein and zeaxanthin are not distributed uniformly, but are excluded from saturated raft domains and about ten times more concentrated in unsaturated bulk lipids. The selective accumulation of lutein and zeaxanthin in direct proximity to unsaturated lipids, which are especially susceptible to lipid peroxidation, could be very important as far as their antioxidant activity is concerned. Therefore, the protective role of lutein against lipid peroxidation was investigated in membranes made of raft-forming mixtures and in models of photoreceptor outer segment membranes and compared with their antioxidant activity in homogeneous membranes composed of unsaturated lipids. Lipid peroxidation was induced by photosensitized reactions using rose Bengal and monitored by an MDA-TBA test, an iodometric assay, and oxygen consumption (using EPR spectroscopy and the MHCTPO spin label as an oxygen probe). The results show that lutein protects unsaturated lipids more effectively in membranes made of raft-forming mixtures than in homogeneous membranes. This suggests that the selective accumulation of macular xanthophylls in the most vulnerable regions of photoreceptor membranes may play an important role in enhancing their antioxidant properties and ability to prevent age-related macular diseases (such as age-related macular degeneration (AMD)).

Key words: antioxidants, macular xanthophylls, lutein, lipid domains, singlet oxygen, AMD

Received: 19 October, 2011; accepted: 01 March, 2012; available on-line: 17 March, 2012

\section{INTRODUCTION}

Xanthophylls lutein and zeaxanthin, which are dipolar carotenoids that possess hydroxyl groups at both ends of the molecule, accumulate at a very high concentration in the macula lutea of the primate eye retina (Snodderly, 1984; Bone et al., 1988; Landrum et al., 1999). The total xanthophyll concentration shows a peak in the central fovea, reaching a value between 0.1 and $1 \mathrm{mM}$ (Landrum et al., 1999). Xanthophylls are mostly located in the Henle's fiber layer of photoreceptor axons (Bone \& Landrum, 1984), but are also found in the membranes of photoreceptor outer segments (POS) (Rapp et al., 2000).

There is substantial evidence that macular xanthophylls can delay age-related vision loss caused by agerelated macular degeneration (AMD) and cataract formation (Gale \& Hall, 2003; Mares, 2004; Christen et al., 2008; Olson et al., 2011). Blue-light filtration (Kirschfeld, 1982) and a direct antioxidant activity (quenching sin- glet oxygen and triplet states of photoreactive molecules and free radical scavenging (Martin et al., 1999; Krinsky et al., 2003) are the mechanisms of the protective action of xanthophylls in the eye. Blue light absorption is considered an indirect antioxidant action because it prevents high-energy blue light from generating reactive oxygen species that can damage photoreceptor cells (Wooten \& Hammond, 2002; Krinsky et al., 2003). Whereas lutein and zeaxanthin present in POS membranes are responsible for protecting membrane lipids (Krinsky et al., 2003), the indirect antioxidant action of macular xanthophylls is associated with their location in the Henle's layer.

The lipid composition of POS membranes (both plas$\mathrm{ma}$ and discs) is known (Boesze-Battaglia \& Schimmel, 1997). They are abundant in long-chain polyunsaturated fatty acids such as docosahexaenoic acid (DHA) that are very susceptible to lipid peroxidation (Beatty et al., 2000; Rapp et al., 2000). The basic lipid composition of POS disc membranes is very similar to that of the raftforming mixture, which usually consists of three types of lipids - unsaturated glycerophospholipids, saturated sphingolipids, and cholesterol - mixed at equimolar concentrations (De Almeida et al., 2003). In this type of mixture, a phase separation takes place: in the liquid-disordered phase, which is rich in unsaturated lipids, rafts are formed that are lipid domains in the liquid-ordered phase consisting mostly of saturated lipids and cholesterol (De Almeida et al., 2003; Subczynski et al., 2007). POS disc membranes contain nearly equimolar concentrations of unsaturated phospholipids (mainly, a polyunsaturated DHA), saturated phospholipids (myristoyl, palmitoyl, and stearoyl), and cholesterol (Boesze-Battaglia \& Schimmel, 1997). Using Triton X-100 extraction at $4^{\circ} \mathrm{C}$ (Ahmed et al., 1997), rafts were isolated as a detergent-resistant fraction (DRM) from POS membranes (Boesze-Battaglia et al., 2002; Martin et al., 2005). The DRM fraction was enriched in saturated lipids and cholesterol, whereas the detergent-soluble fraction (DSM) was rich in DHA (Martin et al., 2005).

Keeping in mind the antioxidant activity of macular xanthophylls in POS membranes on one hand and the domain structure of POS membranes on the other, it seemed very interesting to us to investigate how xantho-

e-mail: anna.m.wisniewska@uj.edu.p

*Presented at the 16th International Symposium on Carotenoids, 17-22 July, 2011, Kraków, Poland

\#Present address: Institute of Physics, Polish Academy of Sciences, Warsaw, Poland

Abbreviations: AMD, age-related macular degeneration; DXPC (where $\mathrm{X}=\mathrm{DHA}, \mathrm{O}, \mathrm{S}$ ), didocosahexaenoyl, dioleoyl, distearoyl, respectively; EPR, electron paramagnetic resonance; MDA, malondialdehyde; PC, phosphatidylcholine; POS, photoreceptor outer segments; RB, rose Bengal; SM, egg sphingomyelin; TBA, tiobarbituric acid. 
phylls are distributed between domains in POS membranes. In our previous work, we showed that in a model of POS membranes, macular xanthophylls are about 14 times more concentrated in the unsaturated bulk domain (enriched in polyunsaturated DHA and isolated as DSM) and excluded from the raft domain (Wisniewska \& Subczynski, 2006a). Similar distribution was found in membranes made of raft-forming mixtures, where lutein and zeaxanthin were about eight times more concentrated in the bulk domain than in the saturated raft domain (Wisniewska \& Subczynski, 2006b). Such a selective accumulation of macular xanthophylls in domains rich in vulnerable unsaturated lipids seems to be ideal for their antioxidant action (Subczynski et al., 2010). Additionally, rhodopsin, the main protein of POS membranes that functions as a visual pigment, is also located in the bulk domain of POS membranes and can be isolated mostly with the DSM fraction (Polozova \& Litman, 2000; Seno et al., 2001; Boesze-Battaglia et al., 2002; Martin et al., 2005). Therefore, the hypothesis was formulated that colocalization of lutein/zeaxanthin, polyunsaturated phospholipids, and rhodopsin may enhance the antioxidant properties of these xanthophylls and be an additional mechanism to prevent oxidative damage to the retina (diseases like AMD).

The aim of the present work was to investigate the protective role of xanthophylls against lipid peroxidation in raft-forming mixtures and in models of photoreceptor membranes and to compare this role with their antioxidant activity in homogeneous membranes composed of unsaturated lipids. To achieve this goal, lipid peroxidation and its inhibition by lutein were studied in two membrane systems with coexisting domains: in well-characterized membranes formed from the raftforming mixture dioleoylphospatidylcholine/sphingomyelin/cholesterol (DOPC/SM/Chol) and in a model of POS membranes consisting of didocosahexaenoylphospatidylcholine/distearoylphospatidylcholine/cholesterol (DHAPC/DSPC/Chol). Both were mixed at a molar ratio of 1:1:1. For comparison, homogeneous membranes made of either DOPC or DHAPC were used.

\section{MATERIALS AND METHODS}

Materials. Lutein was purchased from CaroteNature (Lupsingen, Switzerland). DOPC, SM (egg sphingomyelin), and cholesterol were obtained from Avanti Polar Lipids, Inc. (Alabaster, AL, USA). DSPC and rose Bengal (RB) came from Sigma (St. Louis, MO, USA), and DHAPC was purchased from NOF Corporation Europe. The spin label 4-protio-3-carbamoyl-2,2,5,5-tetraperdeuteromethyl-3-pyrrolin-1-yloxy (mHCTPO) was a generous gift from Prof. Howard J. Halpern at the University of Chicago. Other chemicals of at least reagent grade were obtained from POCH (Gliwice, Poland).

Preparation of liposomes. Model membranes used in this work were multilamellar liposomes obtained by the method of Kusumi and colleagues (Kusumi et al., 1986). Chloroform solutions of lipids and lutein were mixed to achieve desired compound concentrations. Specifically, in the case of the iodometric assay, $6.65 \mu \mathrm{mol}$ of DOPC was used to form homogeneous membranes and 6.65 $\mu \mathrm{mol}$ of each lipid (DOPC, SM, and Chol) was used to form the raft-forming mixture; $1.66 \mu \mathrm{mol}$ of DOPC and $1.66 \mu \mathrm{mol}$ of each lipid in the raft-forming mixtures were added in EPR oximetry experiments; and $2 \mu \mathrm{mol}$ of DHAPC and $2 \mu \mathrm{mol}$ of each lipid (DHAPC, DSPC, and Chol) in the POS membrane model were used in the MDA-TBA test. Therefore, the amount of peroxidizable lipid was always the same in respective homogeneous and heterogeneous membranes, and the molar ratio of individual lipids was always 1:1:1 in a heterogeneous sample. Lutein was added in different concentrations and expressed as $\mathrm{mol} \%$ (relative to total lipids). After the lipids were mixed, the chloroform was evaporated with a stream of nitrogen, and the lipid film was additionally dried under a vacuum for $12 \mathrm{~h}$. Multilayer liposomes were then formed spontaneously by the process of shaking in $2 \mathrm{ml}$ (for iodometric and TBA-MDA tests) or in $0.5 \mathrm{ml}$ (for EPR experiments) of phosphate-buffered saline $(\mathrm{pH} 7.4)$ at a temperature around $60^{\circ} \mathrm{C}$. All preparations with lutein were performed in darkness or dim light and, where possible, under argon.

Irradiation of liposome samples. The process of lipid peroxidation was induced by visible light irradiation. RB was added as a photosensitizer to liposome dispersions just before irradiation at a final concentration of $20 \mu \mathrm{M}$. For iodometric and MDA-TBA assays, irradiation was carried out using a xenon lamp (Muller Elektronic Optik LAX type 1530). The light intensity was 1.5 $\mathrm{mW} / \mathrm{cm}^{2}$. For EPR measurements, irradiation was performed directly in the spectrometer cavity in a flat quartz cell (closed-chamber method) using a Perkin Elmer HX1 lamp (300 W).

Iodometric assay. For the iodometric assay analysis, liposome samples were irradiated up to $15 \mathrm{~min}$ at $12^{\circ} \mathrm{C}$. At higher temperatures, lipid hydroperoxides are unstable. Dispersions of $150 \mu \mathrm{l}$ of liposome were taken at regular intervals during irradiation. Chloroform-methanol $(2: 1, \mathrm{vol} / \mathrm{vol})$ was then added and lipids were extracted according to the procedure described by Folch and colleagues (Folch et al., 1957). The iodometric test was subsequently performed according to the method of Cheeseman (Cheeseman, 1990). The total concentration of accumulated lipid hydroperoxides was calculated based on the absorption of $\mathrm{I}_{3}{ }^{-}$at $353 \mathrm{~nm}$ and assuming the molar extinction coefficient of $2.25 \times 10^{4} \mathrm{M}^{-1} \mathrm{~cm}^{-1}$.

Oxygen photo-consumption. Oxygen consumption during liposome irradiation (up to $15 \mathrm{~min}$ at room temperature) was measured using a Bruker EMX spectrometer operating at $9 \mathrm{GHz}$ and $\mathrm{mHCTPO}$ as a spin probe sensitive to oxygen concentration (Sarna et al., 1980; Wrona et al., 2003). EPR spectra were recorded every $30 \mathrm{~s}$.

MDA-TBA test. MDA-TBA test is based on the detection of malonyldialdehyde (MDA), which is a product of lipid peroxidation. Its reaction with thiobarbituric acid (TBA) at high temperatures produces a colored product (MDA-TBA adduct) with an absorption maximum at $532 \mathrm{~nm}$ (Halliwell \& Chirico, 1993). Irradiation was carried out at two temperatures, $12^{\circ} \mathrm{C}$ and $40^{\circ} \mathrm{C}$, for up to $5 \mathrm{~min}$. Samples were taken every $30 \mathrm{~s}$, and subsequent reaction was stopped by freezing in liquid nitrogen (Broniowska et al., 2007). The concentration of the MDATBA adduct was determined based on the absorbance at $532 \mathrm{~nm}$ and assuming the molar extinction coefficient of $1.56 \times 10^{5} \mathrm{M}^{-1} \mathrm{~cm}^{-1}$.

\section{RESULTS AND DISCUSSION}

\section{Lipid peroxidation in the presence of lutein - lodometric assay}

The inhibition of RB-photosensitized lipid peroxidation by lutein has been studied in DOPC liposomes containing $0,0.1$, and $0.3 \mathrm{~mol} \%$ lutein. $\mathrm{RB}$ is an efficient 

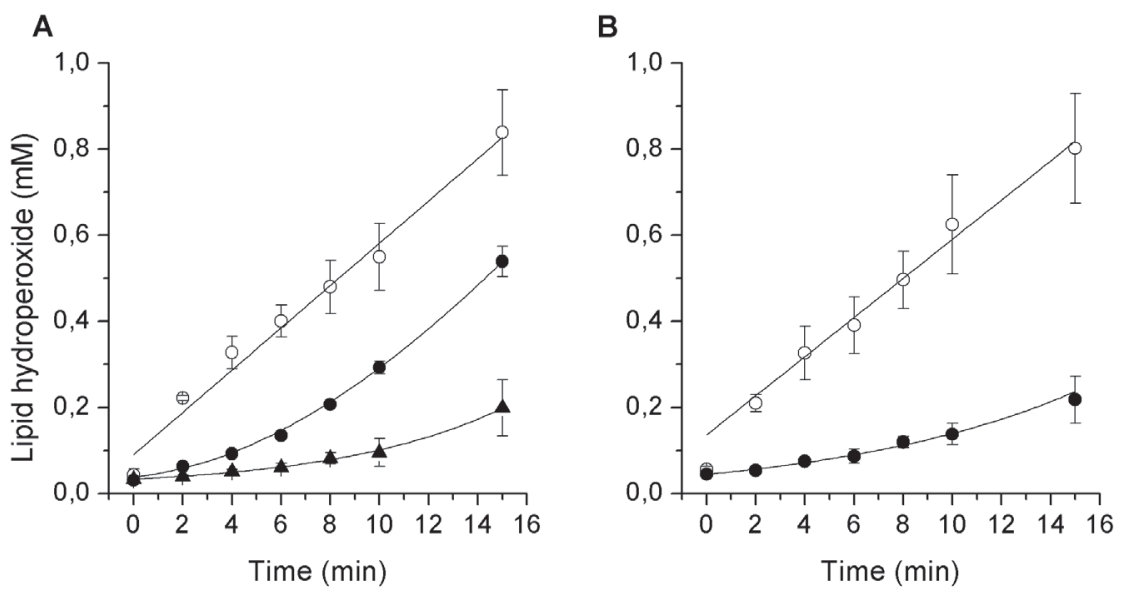

Figure 1. Kinetics of the accumulation of lipid hydroperoxides during DOPC (A) and DOPC/ $\mathrm{SM} / \mathrm{Chol}(\mathrm{B})$ liposome irradiation in the presence of $20 \mu \mathrm{M}$ RB measured by the iodometric assay.

The membranes contained $0(\bigcirc), 0.1(\mathbf{O})$, or $0.3(\mathbf{\Delta})$ mol\% lutein.

photosensitizer, which under aerobic conditions generates singlet oxygen. In turn, singlet oxygen reacts with unsaturated lipids to produce lipid hydroperoxides. Figure $1 \mathrm{~A}$ shows that in the absence of lutein, the lipid hydroperoxide concentration, as detected by the iodometric assay, linearly increases with the time of liposome irradiation. Clearly, in the presence of lutein, the rate of hydroperoxide accumulation slows down, especially during the first minutes of irradiation. Additionally, the effect of lutein is concentration-dependent. This means that in this range of concentration, lutein is dissolved in the membrane and not aggregated. Indeed, data in the literature show that the solubility threshold of dipolar carotenoids in model membranes is 5 to $10 \mathrm{~mol} \%$ (Gabrielska \& Gruszecki, 1996; Subczynski et al., 2010). Also, it was shown that in this range of concentrations, no saturation effect is observed, as far as singlet-oxygen-quenching by xanthophylls in membranes is concerned (Cantrell et al., 2003). A similar experiment was performed for membranes made of raft-forming mixtures of DOPC/SM/ Chol (at a ratio of $1: 1: 1$ ) containing $0.1 \%$ lutein. The results are shown in Fig. 1B. Again, the lipid hydroperoxide concentration increases with the time of liposome irradiation. The inhibition effect of $0.1 \mathrm{~mol} \%$ lutein is significant as well.

To compare the effects of lutein in homogeneous membranes and membranes made of raft-forming mix-

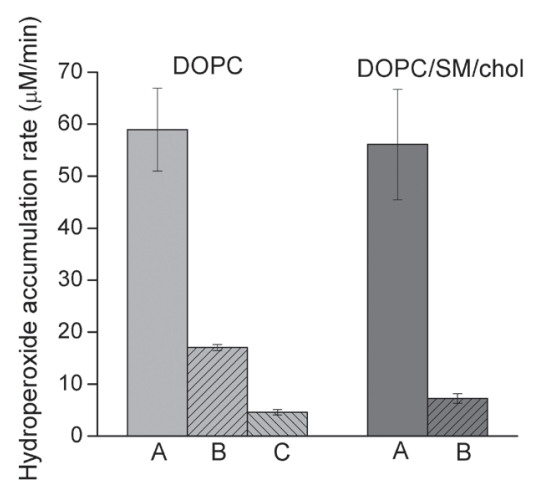

Figure 2. The rate of lipid hydroperoxide accumulation evaluated during the first $6 \mathrm{~min}$ of DOPC and DOPC/SM/Chol liposome irradiation in the presence of $20 \mu \mathrm{M}$ RB.

Membranes contained 0 (A), 0.1 (B), or 0.3 (C) mol\% lutein. Results are the mean \pm S.D. of at least three replicates. tures, an initial hydroperoxide accumulation rate was calculated for the first $6 \mathrm{~min}$ of liposome irradiation and is presented in Fig. 2 as a histogram. It can be seen here that in the absence of lutein the rate of hydroperoxide accumulation is similar in both homogeneous membranes and those made of raft-forming mixtures. This means that the presence of raft domains does not affect the peroxidation process and that the major constituents of raft domains (SM and cholesterol) are not a significant source of additional hydroperoxides. In fact, Wrona et al. (Wrona et al., 2003), using the HPLC-EC technique for separation and quantification of lipid hydroperoxides, showed that the main source of hydroperoxides in liposomes made of ternary lipid mixture (1-palmitoyl-2-oleoyl-PC, dimyristoyl-PC, and cholesterol) and irradiated in the presence of $\mathrm{RB}$ is an unsaturated phospholipid. Cholesterol hydroperoxides are produced with much less efficiency. Figure 2 also clearly shows that lutein significantly reduces the hydroperoxide accumulation rate in both types of membranes. However, the effect is stronger in raft-forming mixtures. Lutein in the concentration as low as $0.1 \mathrm{~mol} \%$ slows down the process of hydroperoxide accumulation by a factor of 3.4 in homogeneous membranes and by a factor of 7.7 in raft-forming mixtures. Obviously, the selective accumulation of lutein in the domain rich in unsaturated lipids (DOPC) helps to prevent peroxidation of the lipid.

\section{Lipid peroxidation in the presence of lutein - oxygen photo-consumption}

During the early stages of lipid peroxidation, molecular oxygen is incorporated in the structure of membrane lipids, and, therefore, its uptake can be used as an indirect measure of peroxidation (Subczynski \& Kusumi, 1985). Also, consumption of oxygen can provide valuable information about the role of antioxidants in this process (Wrona et al., 2003). The oxygen photo-consumption rate was measured for homogeneous DOPC membranes as

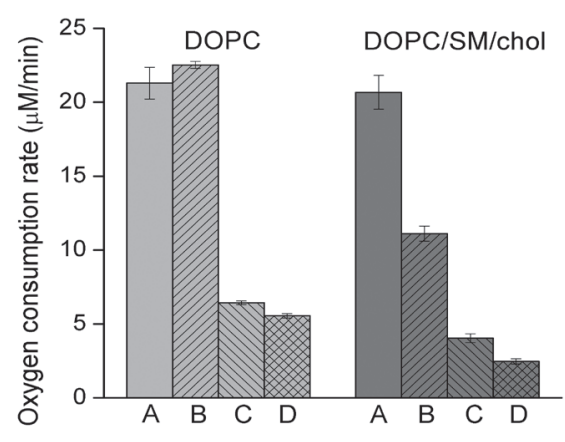

Figure 3. Oxygen consumption rate during the first $6 \mathrm{~min}$ of $\mathrm{DOPC}$ and DOPC/SM/Chol liposome irradiation in the presence of $20 \mu \mathrm{M}$ RB.

Membranes contained 0 (A), 0.1 (B), 0.3 (C), or 0.5 (D) mol\% lutein. Results are the mean \pm S.D. of at least three replicates. 

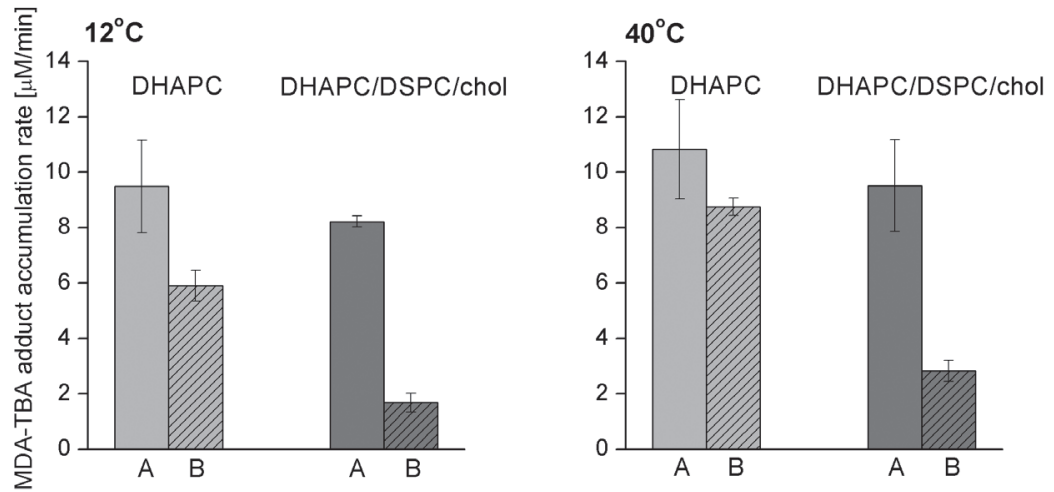

Figure 4. MDA-TBA adduct accumulation rate during DHAPC and DHAPC/DSPC/Chol liposome irradiation at $12^{\circ} \mathrm{C}$ and $40^{\circ} \mathrm{C}$ in the presence of $20 \mu \mathrm{M}$ RB.

Membranes contained: 0 (A) or 0.5 (B) mol\% lutein. Results are the mean \pm S.D. of at least three replicates.

well as for raft-forming mixtures containing DOPC/SM/ Chol at a ratio of 1:1:1 with various lutein concentrations $(0.1,0.3$, and $0.5 \mathrm{~mol} \%$ ). Control experiments were performed in darkness, and no oxygen consumption was detected. Figure 3 shows the initial oxygen consumption rates in all membrane types obtained during the first 6 min of liposome irradiation. Oxygen consumption rates do not differ in homogeneous membranes or in membranes made of raft-forming mixtures where no lutein is present. However, the presence of lutein slows down the process of oxygen consumption significantly, especially at high lutein concentrations $(0.3$ and $0.5 \mathrm{~mol} \%)$. Lutein at a concentration of $0.3 \mathrm{~mol} \%$ inhibits oxygen photo-consumption 3.3 times in DOPC membranes and over 5 times in DOPC/SM/Chol membranes. At a concentration of $0.5 \mathrm{~mol} \%$, the inhibitory effect of lutein is stronger: the oxygen uptake rate is slowed down by a factor of 3.8 in DOPC membranes and by a factor of 8.4 in DOPC/SM/Chol membranes. Like in the case of hydroperoxide accumulation described above, the inhibitory effect of lutein is concentration-dependent. Interestingly, lutein at the lowest concentration used $(0.1 \mathrm{~mol} \%)$ has no effect on oxygen uptake in DOPC membranes but inhibits (nearly two times) oxygen consumption in $\mathrm{DOPC} / \mathrm{SM} / \mathrm{Chol}$ membranes. This result differs from that obtained by the iodometric assay (Fig. 2). Generally, however, the results of the oxygen consumption experiment confirm those of hydroperoxide accumulation obtained by the iodometric assay and support the important role of domain structure in enhancing the antioxidant function of lutein in membranes.

\section{Lipid peroxidation in the presence of lutein - MDA-TBA test}

Another membrane system used in our experiments was the POS membrane model. Lipid peroxidation in membranes made of the equimolar mixture of DHAPC, DSPC, and cholesterol was compared with that in homogeneous membranes made of DHAPC only. In the case of this model, an MDA-TBA test was used as a convenient measure of lipid peroxidation (taking advantage of the presence of polyunsaturated lipids). Additionally, since MDA is more stable at higher temperatures than lipid hydroperoxides, RB-photosensitized lipid peroxidation was also performed at $40^{\circ} \mathrm{C}$. The results presented in Fig. 4 show a general increase in MDA-TBA adduct accumulation at $40^{\circ} \mathrm{C}$ compared with that at $12^{\circ} \mathrm{C}$ in both homogeneous membranes and membranes made of raft-forming mixtures and in both the absence and pres- ence of $0.5 \mathrm{~mol} \%$ lutein. In contrast to results obtained by the iodometric assay and EPR oximetry, the MDATBA test shows a difference between the amounts of peroxidation in homogeneous and heterogeneous membranes in the absence of lutein. At both temperatures, the MDATBA accumulation rates in homogeneous membranes are about $13 \%$ greater than in membranes made of raft-forming mixtures. However, the antioxidant effect of lutein on both types of membranes is similar to that observed previously: it is clearly stronger in membranes made of raftforming mixture than in homogeneous membranes. At $12^{\circ} \mathrm{C}, 0.5 \mathrm{~mol} \%$ lutein decreases the MDA-TBA accumulation rate by a factor of 1.6 in DHAPC membranes and by a factor of 4.8 in DHAPC/ DSPC/Chol membranes. Similarly, at $40^{\circ} \mathrm{C}$, the MDATBA accumulation rate is reduced by a factor of 1.2 in DHAPC and by a factor of 3.4 in DHAPC/DSPC/Chol membranes. This shows that the effects of lutein are generally weaker at $40^{\circ} \mathrm{C}$, which may result from partial degradation of the xanthophyll at higher temperatures.

\section{CONCLUSION}

Carotenoids may act as antioxidants by three mechanisms: quenching singlet oxygen, quenching excited states of photosensitizers, and scavenging free radicals (Krinsky et al., 2003). The first mechanism is especially important since lutein and especially zeaxanthin are very effective quenchers of singlet oxygen. Their bimolecular rate constants of singlet-oxygen quenching in solution are in the range of $6.6-16 \times 10^{9} \mathrm{M}^{-1} \mathrm{~s}^{-1}$ (Cantrell et al., 2003). The retina is very sensitive to oxidative stress and lipid peroxidation because it is abundantly illuminated, has high respiratory demands for oxygen, and contains a high concentration of polyunsaturated lipids, mainly DHA (Beatty et al., 2000). Also, endogenous photosensitizers, such as lipofuscin or retinal, may produce singlet oxygen (Rozanowska \& Sarna, 2005). This means that retina membranes need to be effectively protected against reactive species; macular xanthophylls serve this function well. Additionally, our results show that their selective accumulation in domains enriched in unsaturated lipids significantly enhances their effectiveness as antioxidants in lipid membranes. In all of the systems studied, lutein was two to three times more effective in membranes containing domains than in homogeneous, unsaturated membranes. Interestingly, the greatest difference between the antioxidant effect of lutein on homogeneous and heterogeneous membranes was observed by the MDA-TBA test in the model of POS membranes (Fig. 4). Moreover, the MDA-TBA test showed that DHAPC is oxidized to a lesser extent when present in domain-structured membranes (such as the model of POS membranes) than in homogeneous membranes, even in the absence of lutein. Because in raft-forming mixtures unsaturated lipids are also present in rafts, it may be suggested that this small fraction of DHAPC is protected by the membrane structure (the raft domain is more rigid than the surrounding bulk domain, and molecules such as oxygen have limited diffusion possibilities) (Wisniewska \& Subczynski, 2006a, 2006b; Subczynski et al., 2007). The results obtained in 
this work confirm our previous hypothesis (Subczynski et al., 2010) that the membrane domain structure plays an important role in protection membrane sensitive molecules by co-localizing them with protective molecules.

\section{Acknowledgements} NIH.

This work was supported by grant EY015526 of the

\section{REFERENCES}

Ahmed SN, Brown DA, London E (1997) On the origin of sphingolipid/cholesterol-rich detergent-insoluble cell membranes: physiological concentrations of cholesterol and sphingolipid induce formation of a detergent-insoluble, liquid-ordered lipid phase in model membranes. Biochemistry 36: 10944-10953.

De Almeida RFM, Fedorov A, Prieto M (2003) Sphingomyelin/phosphatidylcholine/cholesterol phase diagram: boundaries and composition of lipid rafts. Biophys J 85: 2406-2416.

Beatty S, Koh HH, Phil M, Henson D, Boulton M (2000) The role of oxidative stress in the pathogenesis of age-related macular degeneration. Surv Opbthalmol 45: 115-134.

Boesze-Battaglia K, Dispoto J, Kahoe MA (2002) Association of a photoreceptor-specific tetraspanin protein, ROM-1, with triton X100-resistant membrane rafts from rod outer segment disk membranes. J Biol Chem 277: 41843-41849.

Boesze-Battaglia K, Schimmel R (1997) Cell membrane lipid composition and distribution: implications for cell function and lessons learned from photoreceptors and platelets. J Exp Biol 200: 29272936.

Bone RA, Landrum JT (1984) Macular pigment in Henle fiber membranes: a model for Haidinger's brushes. Vision Res 24: 103-108.

Bone RA, Landrum JT, Fernandez L, Tarsis SL (1988) Analysis of the macular pigment by HPLC: retinal distribution and age study. Invest Ophthalmol Vis Sci 29: 843-849.

Broniowska KA, Kirilyuk I, Wisniewska A (2007) Spin-labelled lutein as a new antioxidant in protection against lipid peroxidation. Free Radic Res 41: 1053-1060.

Cantrell A, McGarvey DJ, Truscott TG, Rancan F, Böhm F (2003) Singlet oxygen quenching by dietary carotenoids in a model membrane environment. Arch Biochem Biophys 412: 47-54.

Cheeseman KA (1990) Methods of measuring lipid peroxidation in biological systems: an overview. In Free Radicals, Lipoproteins and Membrane Lipids. Crastes de Paulet A, Douste-Blazy L, Raoletti R, eds, pp 143-152. Plenum Press, New York.

Christen WG, Liu S, Glynn RJ, Gaziano JM, Buring JE (2008) Dietary carotenoids, vitamins $\mathrm{C}$ and $\mathrm{E}$, and risk of cataract in women: a prospective study. Arch Ophthalmol 126: 102-109.

Folch J, Lees M, Sloane-Stanley G, others (1957) A simple method for the isolation and purification of total lipids from animal tissues. J Biol Chem 226: 497-509.

Gabrielska J, Gruszecki WI (1996) Zeaxanthin (dihydroxy-[beta]-carotene) but not [beta]-carotene rigidifies lipid membranes: a 1H-NMR study of carotenoid-egg phosphatidylcholine liposomes. Biochim Biophys Acta 1285: 167-174.

Gale C, Hall N (2003) Lutein and zeaxanthin status and risk of age-related macular degeneration. Invest Ophthalmol Vis Sci 44: 2461-2465.

Halliwell B, Chirico S (1993) Lipid peroxidation: its mechanism, measurement, and significance. Am J Clin Nutr 57: 715S-724S.

Kirschfeld K (1982) Carotenoid pigments: their possible role in protecting against photooxidation in eyes and photoreceptor cells. Proc $\mathrm{R}$ Soc Lond B Biol Sci 216: 71-85.
Krinsky NI, Landrum JT, Bone RA (2003) Biologic mechanisms of the protective role of lutein and zeaxanthin in the eye. Annu Rev Nutr 23: 171-201.

Kusumi A, Subczynski WK, Pasenkiewicz-Gierula M, Hyde JS, Merkle H (1986) Spin-label studies on phosphatidylcholine-cholesterol membranes: effects of alkyl chain length and unsaturation in the fluid phase. Biochim Biophys Acta 854: 307-317.

Landrum J, Bone R, Moore LL, Gomea CM (1999) Analysis of zeaxanthin distribution within individual human retinas. Methods Ensymol 229: $457-467$.

Mares J (2004) Carotenoids and eye disease: epidemiological evidence. In Carotenoids in health and disease. Dekker M, ed, pp 427-444. Marcel Dekker, New York.

Martin H, Ruck C, Schmidt M, Sell S, Beutner S, Mayer B, Walsh R (1999) Chemistry of carotenoid oxidation and free radical reactions. Pure Appl Chem 71: 2253-2262.

Martin RE, Elliott MH, Brush RS, Anderson RE (2005) Detailed characterization of the lipid composition of detergent-resistant membranes from photoreceptor rod outer segment membranes. Invest Ophthalmol Vis Sci 46: 1147-1154.

Olson JH, Erie JC, Bakri SJ (2011) Nutritional Supplementation and Age-Related Macular Degeneration. In Semin Ophthalmol. pp 131136. Informa Healthcare, New York.

Polozova A, Litman BJ (2000) Cholesterol dependent recruitment of di22: 6-PC by a $G$ protein-coupled receptor into lateral domains. Biophys J 79: 2632-2643.

Rapp LM, Maple SS, Choi JH (2000) Lutein and zeaxanthin concentrations in rod outer segment membranes from perifoveal and peripheral human retina. Invest Ophthalmol Vis Sci 41: 1200-1209.

Rozanowska M, Sarna T (2005) Light-induced damage to the retina: role of rhodopsin chromophore revisited. Photochem Photobiol 81: $1305-1330$.

Sarna T, Duleba A, Korytowski W, Swartz H (1980) Interaction of melanin with oxygen. Arch Biochem Biophys 200: 140-148.

Seno K, Kishimoto M, Abe M, Higuchi Y, Mieda M, Owada Y, Yoshiyama W, Liu H, Hayashi F (2001) Light-and guanosine 5'-3-O-(thio) triphosphate-sensitive localization of a $G$ protein and its effector on detergent-resistant membrane rafts in rod photoreceptor outer segments. I Biol Chem 276: 20813-20816.

Snodderly D (1984) The macular pigment. II. Spatial distribution in primate retinas. Invest Ophthalmol V is Sci 25: 674-685.

Subczynski WK, Kusumi A (1985) Detection of oxygen consumption during very early stages of lipid peroxidation by ESR nitroxide spin probe method. Biochim Biophys Acta 821: 259-263.

Subczynski WK, Wisniewska A, Hyde JS, Kusumi A (2007) Three-dimensional dynamic structure of the liquid-ordered domain in lipid membranes as examined by pulse-EPR oxygen probing. Biophys $J$ 92: 1573-1584.

Subczynski WK, Wisniewska A, Widomska J (2010) Location of macular xanthophylls in the most vulnerable regions of photoreceptor outer-segment membranes. Arch Biochem Biophys 504: 61-66.

Wisniewska A, Subczynski WK (2006a) Distribution of macular xanthophylls between domains in a model of photoreceptor outer segment membranes. Free Radic Biol Med 41: 1257-1265.

Wisniewska A, Subczynski WK (2006b) Accumulation of macular xanthophylls in unsaturated membrane domains. Free Radic Biol Med $\mathbf{4 0}$ : 1820-1826.

Wooten BR, Hammond BR (2002) Macular pigment: influences on visual acuity and visibility. Prog Retin Eye Res 21: 225-240.

Wrona M, Korytowski W, Rozanowska M, Sarna T, Truscott TG (2003) Cooperation of antioxidants in protection against photosensitized oxidation. Free Radic Biol Med 35: 1319-1329. 
